\title{
Singular continuous spectra in a pseudo-integrable billiard
}

\author{
Jan Wiersig \\ Max-Planck-Institut für Physik komplexer Systeme,D-01187 Dresden, Germany
}

(October 27, 2018)

\begin{abstract}
The pseudo-integrable barrier billiard invented by Hannay and McCraw [J. Phys. A 23, 887 (1990)] - rectangular billiard with line-segment barrier placed on a symmetry axis - is generalized. It is proven that the flow on invariant surfaces of genus two exhibits a singular continuous spectral component.
\end{abstract}

PACS number: 05.45.-a

Billiards have attracted much attention in the recent decades as simple dynamical systems in classical and quantum mechanics. The free motion of a particle in a domain within a hard elastic boundary shows a large spectrum of behaviour depending on the shape of the boundary, ranging from complete regularity to various degrees of chaoticity.

Polygonal billiards are neither chaotic - the Kolmogorov-Sinai entropy is zero - nor integrable in the sense of Liouville-Arnol'd [1] (apart from the rectangles, the equilateral triangles, the $\pi / 2, \pi / 4, \pi / 4$-triangles and the $\pi / 2, \pi / 3, \pi / 6$-triangles). The motion inside a typical polygon is conjectured to be ergodic on the three-dimensional constant-energy surfaces in phase space, while the motion inside a rational polygon (all angles are rationally related to $\pi$ ) is restricted to twodimensional invariant surfaces, like in integrable systems, but the genus of the surfaces is larger than 1. Rational polygonal billiards are therefore characterized as pseudointegrable [2]. It is rigorously proven that the flow on these surfaces is ergodic and not mixing [3]. Ergodicity means that a particle with a typical initial momentum explores the entire invariant surface (which is the billiard table in the configuration space projection). Since the mixing property is excluded, a small cluster of particles with identical initial momenta cannot spread out uniformly in time on the invariant surface. But a nonuniform spreading which allows occasional reclustering with decreasing frequency - weak mixing in mathematical terms (see e.g. 幽) - is possible. In fact, weak mixing is believed to be generic, but this is only shown for polygons all of whose sides are horizontal or vertical. Numerical evidence for weak mixing has been reported for the "square-ring billiard" 5]; see also related numerical studies on rational [6] and irrational triangles [7,8].

Weak mixing (in the absence of the stronger mixing property) implies interesting spectral properties [9]. More precisely, the Fourier transform of a typical function on the phase space with respect to time has a fractallike structure in the frequency domain. Such a spectrum is characterized as singular continuous, in contrast to discrete spectra of integrable systems (nonmixing, quasiperiodic motion) and absolutely continuous spectra of chaotic systems (mixing motion). Singular continu- ous spectra are well known in other fields of physics to appear at the border between integrability and chaos, e.g. strange nonchaotic attractors (see, e.g., [10,11]), the (kicked) Harper model 12 and models for nonperiodic atomic structures (see [13] and references therein).

The spectral properties of a phase space function are related to its autocorrelation function $(\mathrm{AF})$. For discrete spectra this function is (quasi-) periodic. In the periodic case the (normalized) AF returns exactly to 1, while in the quasiperiodic case it comes arbitrarily close to 1 . An exponentially decreasing AF (for increasing time) indicates an absolutely continuous spectrum. An AF corresponding to a singular continuous spectrum does not usually decay to zero, but also does not return to 1 .

A nongeneric subclass of pseudo-integrable systems is given by almost-integrable billiards [14]. A member of this class is, roughly speaking, composed of several copies of a single completely integrable billiard, e.g. the $\pi / 3,2 \pi / 3$-rhombus 15] consists of two identical equilateral triangles. Motion on invariant surfaces of almostintegrable billiards is not weakly mixing 14]. The aim of this letter is to establish that the spectra of these systems nevertheless can have a singular continuous component (together with a discrete component). We prove this for a simple model, a generalized version of the "barrier billiard" [16]. It is the first time that the existence of singular spectra in a given billiard is rigorously shown. This is achieved by decomposing the dynamics into a continuous and a discrete part: the quasiperiodical motion in the integrable subbilliard and the transitions between different copies. The latter type of motion, responsible for the singular continuous component, is discussed with the help of the AF.

We consider a point particle with unit mass elastically bouncing inside a polygonal enclosure consisting of a vertical line of length $1-\beta \in(0,1)$ symmetrically placed in a rectangle with width $L \in(0, \infty)$ and normalized height 1, see Fig. 1(a). The special case $\beta=1 / 2$ corresponds to the usual barrier billiard [16]. The trivial energy dependence of the system is ignored by setting the energy to $1 / 2$, or equally the magnitude of the momentum $\left(p_{x}, p_{y}\right)$ to 1 . Given an initial momentum only a finite number of directions is realized during the time evolution, due to the fact that all angles in the polygon are rational mul- 
tiples of $\pi$. In other words, the motion in phase space takes place on two-dimensional invariant surfaces. Using the general formula for the genus of such surfaces [2] gives 2 , i.e. the surfaces have the topology of two-handled spheres and not that of tori (single-handled spheres).
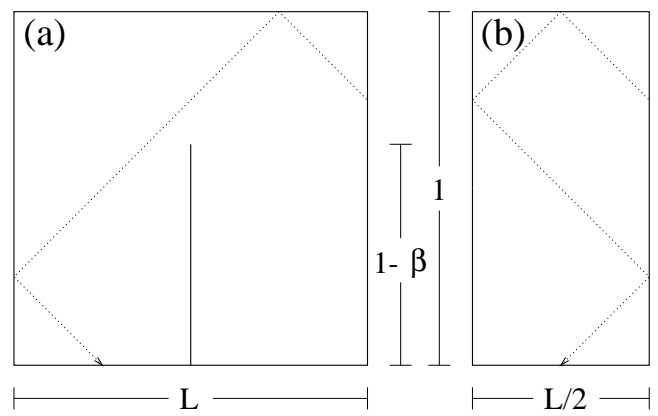

FIG. 1. (a) Barrier billiard, rectangle with a vertical line connecting the origin of the coordinate system $(x, y)=(0,0)$ with the point $(x, y)=(0,1-\beta)$. (b) Symmetry reduced version.

Our model satisfies the definition of almostintegrability given by Gutkin [14 for all values of $L$ and $\beta$. It is composed of two copies of an integrable rectangular billiard, which can be obtained by symmetry reduction, see Fig. 1 (b). The dynamics can be split into two parts. First, the quasiperiodical dynamics $(|x(t)|, y(t))$ on the invariant tori of the symmetry reduced system. Second, the dynamics of the sign of $x$, $s(t)= \pm 1$.

The quasiperiodical component is most elegantly described in action-angle variables. The actions $I_{x}=$ $\left|p_{x}\right| L / 2 \pi$ and $I_{y}=\left|p_{y}\right| / \pi$ label the invariant tori while the angles (divided by $2 \pi$ and computed modulo 1 ) describe the flow on a given torus

$$
\begin{array}{ll}
\phi_{x}(t)=\frac{\omega_{x}}{2 \pi} t+\phi_{x, o} & (\bmod 1) \\
\phi_{y}(t)=\frac{\omega_{y}}{2 \pi} t+\phi_{y, o} & (\bmod 1),
\end{array}
$$

with the frequencies $\omega_{x}=4 \pi^{2} I_{x} / L^{2}$ and $\omega_{y}=\pi^{2} I_{y}$. The flow on the torus is ergodic if and only if the winding number $\rho=\omega_{y} / \omega_{x}=I_{y} L^{2} /\left(4 I_{x}\right)$ is irrational. The motion in configuration space is explicitly given by

$$
\begin{aligned}
|x(t)| & =\frac{L}{2} f\left(\phi_{x}(t)\right) \\
y(t) & =f\left(\phi_{y}(t)+\frac{1-\beta}{2} \quad(\bmod 1)\right)
\end{aligned}
$$

with the piece-wise linear function

$$
f(u)=\left\{\begin{array}{cl}
2 u & \text { if } 0 \leq u<1 / 2 \\
2(1-u) & \text { otherwise }
\end{array}\right.
$$

The shift in the argument of the function $f$ in Eq. (4) will be convenient later.
Now we consider the non-quasi-periodical part of the dynamics. Clearly, the value of $s(t)$ changes when the trajectory impinges on the segment $\{x=0$ and $1-\beta<y\}$ (or $\left\{\phi_{x}=0\right.$ and $\left.0<\phi_{y}<\beta\right\}$ ). We therefore introduce the Poincaré section $\phi_{x}(n T)=0$ with $T=2 \pi / \omega_{x}$ and the integer $n$. We obtain

$$
\begin{aligned}
\phi_{y, n+1} & =\phi_{y, n}+\rho \quad(\bmod 1) \\
s_{n+1} & =s_{n} \Phi\left(\phi_{y, n}\right)
\end{aligned}
$$

with

$$
\Phi(\phi)=\left\{\begin{array}{cl}
-1 & \text { if } 0<\phi<\beta \\
1 & \text { otherwise }
\end{array}\right.
$$

Note that diffractive orbits, i.e. orbits which hit the edge of the barrier, can be neglected since they have measure zero in phase space. The two-dimensional mapping (5)(6) has the solution

$$
s_{n}=s_{0} \Pi_{j=0}^{n} \Phi\left(\phi_{y, 0}+j \rho(\bmod 1)\right) .
$$

This equation together with Eq. (11)-(位) and $n$ given by the integer part of $t / T$ solve the billiard problem. But the solution is only formal, because in order to compute (7) one has to iterate (5)-(6). A similar, but more complicated, solution of the rhombus billiard was reported in [15]. But its formality was not recognized since the authors focussed only on the equation corresponding to (5), while the equation corresponding to (6) was hidden in the notation.

Having defined the dynamical system, we now discuss the spectral properties of a typical smooth phase space function $F\left(x, p_{x}, y, p_{y}\right)$ (nontypical functions $F\left(x^{2}, p_{x}^{2}, y, p_{y}\right)$ obviously have discrete spectra). We investigate firstly the function $F(t)=x(t)$ (the argumentation for $p_{x}(t)$ is identical). We will see that $x(t)$ has a singular continuous spectrum, which implies that a typical function has a mixture of discrete and singular continuous spectrum. With $x(t)=s(t)|x(t)|$ it is clear that the Fourier transform of $x(t)$,

$$
X(\omega)=\int_{-\infty}^{\infty} x(t) e^{-i \omega t} d t
$$

can be written as convolution of the Fourier transforms of $|x(t)|$ and $s(t), \bar{X}(\omega)$ and $S(\omega)$

$$
X(\omega)=\int_{-\infty}^{\infty} \bar{X}\left(\omega^{\prime}\right) S\left(\omega-\omega^{\prime}\right) d \omega^{\prime} .
$$

Since $|x(t)|$ is periodic with period $T$ we have

$$
\bar{X}\left(\omega^{\prime}\right)=\sum_{m=-\infty}^{\infty} A_{m} \delta\left(\omega^{\prime}-m \omega_{x}\right) .
$$

Substituting into Eq. (9) gives 


$$
X(\omega)=\sum_{m=-\infty}^{\infty} A_{m} S\left(\omega-m \omega_{x}\right)
$$

Since $s(t)$ changes in discrete steps it is suitable to consider the discrete-time Fourier transform of the sequence $\left[s_{n}\right]$

$$
\Sigma(\omega)=\sum_{n=-\infty}^{\infty} s_{n} e^{-i \omega n}
$$

which is related to the continuous-time Fourier transform via

$$
S(\omega)=\frac{1}{i \omega}\left(1-e^{i \omega T}\right) \Sigma(\omega T)
$$

Note that the prefactor goes to the constant $T$ as $\omega \rightarrow 0$. We can now take advantage of the fact that the mapping (5)-(6) is well studied. It is proven (see [17] and references therein) that if $\rho$ is irrational then $\Sigma(\omega)$ is singular continuous for almost all $\beta$ (the spectrum is discrete, e.g., if $\beta=n \rho(\bmod 1))$. Since this holds trivially also for any nonconstant function of $s(t)$ we conclude that the $s$-motion is weakly mixing 9]. The fact that almost all trajectories in our billiard have irrational winding number $\rho$, independent of the values of the parameters $L$ and $\beta$, ensures that typical trajectories give rise to singular spectra $\Sigma(\omega)$. Together with Eq. (10) and (11) this implies that $X(\omega)$ is typically singular continuous. Nevertheless, there exist nonconstant functions of $x(t)$, e.g. $x^{2}(t)$, which do not have a singular continuous spectrum, so the $x$-motion cannot be weakly mixing. This result indicates that a purely numerical detection of weak mixing by investigating the spectral properties of just a single phase space variable may lead to wrong conclusions.

Figure 2 shows an example of $|X(\omega)|^{2}$ close to the frequency $\omega_{x}$ (so essentially $\left|S\left(\omega-\omega_{x}\right)\right|^{2}$ is shown) for the case $\beta=1 / 2$ and $\rho$ equal the reciprocal of the golden mean, $\rho_{\mathrm{gm}}=(\sqrt{5}-1) / 2$. The integration (8) has been performed with a fading factor $\exp \left(-2 t / t_{\max }\right)$ and truncated at $t_{\max }=10000$. The enlargement in Fig. 2 $\mathrm{re}$ veals some selfsimilarity of $S(\omega)$ for this set of parameters. The minor lack of symmetry is due to the prefactor in Eq. (11); $\Sigma(\omega)$ is perfectly selfsimilar as shown by Feudel et al. [11] by using a renormalization group approach (compare the magnification in Fig. 2 with Fig. 3 in [11). Feudel et al. have also calculated the generalized dimensions: (i) the Hausdorff dimension $D_{0}=1$, i.e. the spectrum is continuous. (ii) The information dimension $D_{1} \approx 0.75$, i.e. the spectrum is singular. (iii) The correlation dimension $D_{2} \approx 0.65$, which is the exponent of the power-law decay $t^{-D_{2}}$ of the integrated AF [18].

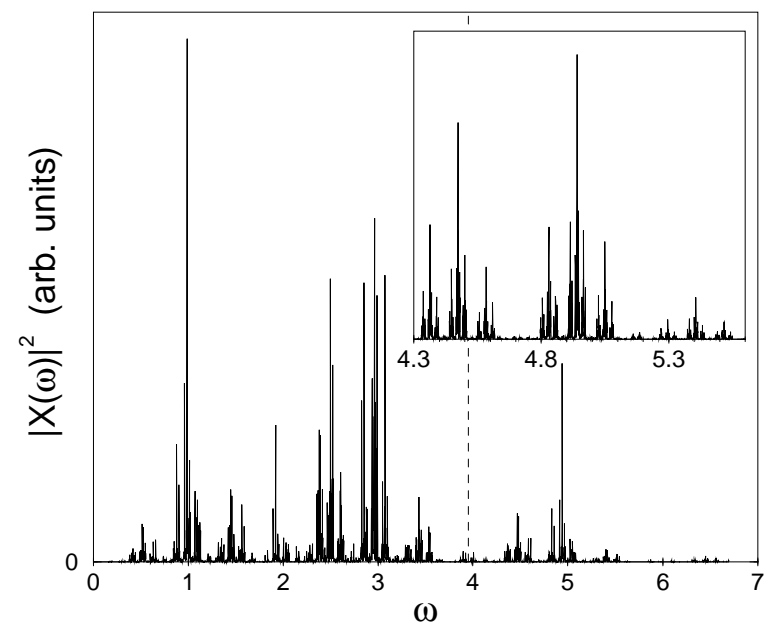

FIG. 2. Power spectrum $|X(\omega)|^{2}$ for $\beta=1 / 2$ and $\rho=\rho_{\mathrm{gm}}$. The dashed line marks the fundamental frequency of $|x(t)|$, $\omega_{x} \approx 3.95187$. The inset shows a magnification.

Before discussing the integrated AF, let us first consider the (normalized) AF $(x(t)$ has zero mean value)

$$
R_{x}(\tau)=\frac{\langle x(t+\tau) x(t)\rangle}{\left\langle x^{2}(t)\right\rangle}
$$

where the average $\langle\ldots\rangle$ is taken over $t$. We restrict ourself to the times $\tau=n T$. By using the periodicity of $|x(t)|$ it is an easy matter to show that $R_{x}(n T)$ is equal to $R_{s}(n)$, the $\mathrm{AF}$ of the process $s_{n}$ (where the average is over the discrete time $n$ ). This means we discuss here the correlations of the transitions between the two copies of the integrable subbilliard. For the special case $\beta=1 / 2$, $R_{s}(n)$ as function of $n$ is studied in 10 for different $\rho$. It is shown that $R_{s}(n)$ vanishes for odd $n$. For large even $n$ it is mostly close to zero but has large peaks at positions which roughly grow exponentially. For example, if $\rho=\rho_{\text {gm }}$ then peaks with amplitude $\approx 0.55$ are located at all even Fibonacci numbers surrounded by smaller peaks in a selfsimilar manner.

We have determined the integrated $\mathrm{AF}$

$$
R_{s, \text { int }}(n)=\frac{1}{n} \sum_{j=0}^{n}\left|R_{s}(j)\right|^{2}
$$

up to $n_{\max }=10000$. Its asymptotic behaviour is found to be in agreement with the expected power-law decay, which allows us to compute $D_{2}$ numerically. For fixed $\rho=\rho_{\mathrm{gm}}, D_{2}$ as function of $\beta$ (using 1000 randomly distributed $\beta$-values) has mean value 0.458 and shows very strong fluctuations, ranging from large decay rates $D_{2}=0.68$ to zero decay. These fluctuations are by no means surprising, since there are dense sets of $\beta$-values (e.g. $\beta=n \rho(\bmod 1))$ for which $D_{2}$ vanishes. We have also calculated $D_{2}$ for fixed $\beta=1 / 2$ as a function of $\rho$ (using $1000 \rho$-values). Here, $D_{2}$ is more or less uniformly distributed between zero and 0.94 around a mean 
value of 0.469 . Due to the sensitive dependence of $D_{2}$ on the winding number, it seems useful to perform an average of the AF over all invariant surfaces (i.e. over all $\rho$ ) in order to characterize the correlation properties of the transitions between the two copies of the subbilliard as a whole. But this kind of averaging does not yield a reasonable correlation function since for an integrable system such a function would indicate correlation decay 19.

The fact that the integrated AF $(13)$ decays to zero but $R_{s}$ does not confirms the weak-mixing property of the $s$-motion. Because of $R_{x}(n T)=R_{s}(n)$ one could naively draw the wrong conclusion that the $x$-motion is also weakly mixing. This confusion can be resolved if one clearly distinguishes between continuous-time and discrete-time approaches. Trivially, $R_{x}$ does not decay for large continuous times $\tau$ if it does not decay for large discrete times $n$. But we expect that the integrated (now really an integration not a summation) AF of $x(t)$ does not decay, in contrast to the integrated AF of the process $s_{n}$ (the integrated AF of a general function $F\left(x, y, p_{x}, p_{y}\right)$ obviously shows zero decay rates, since $y(t)$ is periodic). This agrees with results of the study on an almost-integrable version of the "square-ring billiard" [5]. It is worth emphasising that the discretetime approach, which is always used in numerical studies where integrations are replaced by summations (for billiards the discrete-time approach is often used explicitly), can wrongly indicate weak mixing, even though this is quite unlikely.

In terms of the billiard motion we have the following scenario. The motion is not mixing and even not weakly mixing; that means, a small cluster of particles with the same initial momenta will not spread out on the entire billiard table. But the motion is "partly weak mixing" in the sense that after some transient time (when the AF is close to zero) there are two clusters, one in the left and one in the right part, each of them consisting of approximately half of the particles. At "resonant" times (corresponding to the peaks of the AF) one of the two clusters contains more particles than the other one. But these reclusterings become more and more rare with increasing time.

To summarize, we have proven that the motion in the pseudo-integrable barrier billiard exhibits a discrete and a singular continuous spectral component. We expect that this is true for all almost-integrable billiards.

I would like to thank R. Artuso for useful comments and M. Sieber and J. Nöckel for discussions.

[1] V. I. Arnold, Mathematical Methods of Classical Mechanics, Vol. 60 of Graduate Texts in Mathematics (Springer,
Berlin, 1978).

[2] P. J. Richens and M. Berry, Physica D2, 495 (1981).

[3] E. Gutkin, J. Stat. Phys. 83, 7 (1996).

[4] V. I. Arnol'd and A. Avez, Ergodic Problems of Classical Mechanics (Benjamin-Cummings, Reading, MA, 1968).

[5] R. Artuso, I. Guarneri, and L. Rebuzzini (unpublished).

[6] R. Artuso, Physica 109D, 1 (1997).

[7] R. Artuso, G. Casati, and I. Guarneri, Phys. Rev. E 55, 6384 (1997).

[8] G. Casati and T. Prosen, Phys. Rev. Lett. 83, 4729 (1999).

[9] I. Cornfeld, S. Fomin, and Y. Sinai, Ergodic Theory (Springer, Berlin, 1982).

[10] A. Pikovsky and U. Feudel, J. Phys. A: Math. Gen. 27, 5209 (1994).

[11] U. Feudel, A. Pikovsky, and A. Politi, J. Phys. A: Math. Gen. 29, 5297 (1996).

[12] R. Artuso and G. Casati, Int. J. of Mod. Phys. B 8, 207 (1994).

[13] C. Godrèche and J. Luck, J. Phys. A: Math. Gen. 23, 3769 (1990).

[14] E. Gutkin, Physica 19D, 311 (1986).

[15] B. Eckhardt, J. Ford, and F. Vivaldi, Physica 13D, 339 (1984).

[16] J. H. Hannay and R. J. McCraw, J. Phys. A: Math. Gen. 23, 887 (1990).

[17] G. W. Riley, J. London Math. Soc. 17, 152 (1978).

[18] R. Ketzmerick, G. Petschel, and T. Geisel, Phys. Rev. Lett. 69, 695 (1992).

[19] G. Casati and F. Valz-Gris, Physica 3D, 644 (1981). 